\title{
Valoración del mecanismo de terminación anticipada en los contratos de concesión 4G en Colombia
}

\section{Valuation of the early termination mechanism in $4 \mathrm{G}$ concession contracts in Colombia}

\author{
Alejandro Giraldo Vásquez*
}

* Profesional en Gobierno y Relaciones Internacionales. Consultor independiente en Evaluación de Proyectos, Project Finance y estructuración de APP. Bogotá (Colombia)

[giraldoalejandro9@gmail.com], [https://orcid.org/0000-0002-0291-0695].

Fecha de recepción: 1 de marzo de 2019.

Fecha de aceptación: 30 de mayo de 2019.

Para citar este artículo:

Giraldo Vásquez, A. (2019). Valoración del mecanismo de terminación anticipada en los contratos de concesión 4G en Colombia. ODEON, 16, 67-95.

DOI: https://doi.org/10.18601/17941113.n16.04 


\section{Resumen}

La terminación anticipada de asociaciones público-privadas (APP) suele ocurrir en cualquier momento durante la vida del proyecto y ocasiona grandes costos para las partes. Particularmente, las concesiones viales se enfrentan a un alto riesgo de demanda. En general, los contratos de concesión vial incorporan un mecanismo de compensación en caso de terminación anticipada. No obstante, la técnica tradicional del flujo de caja descontado (FCD), utilizada para valorar proyectos de infraestructura, no captura la irreversibilidad de la inversión, la incertidumbre y los mecanismos de compensación por riesgo que permiten tomar acciones a las partes intervinientes de un contrato de concesión. La terminación anticipada de una concesión vial puede ser valorada como el derecho que tiene el concesionario a abandonar la ejecución del proyecto. El objetivo del presente documento es valorar el mecanismo de terminación anticipada de una concesión vial como un activo contingente, implementando la teoría de las opciones reales, donde el valor de la opción de abandono depende del riesgo de demanda. La metodología de valoración es aplicada a un estudio de caso real del programa de la Cuarta Generación de Concesiones Viales del Gobierno de Colombia. La valoración de los mecanismos de terminación anticipada puede ayudar a comprender de manera ex-ante los riesgos a los cuales se enfrentan las partes pública y privada de un contrato de concesión.

Palabras clave: opciones reales; mecanismos de compensación; terminación anticipada; asociaciones público-privadas.

Clasificación JEL: G13, D86, H43, H54.

\section{Abstract}

The early termination of Public-Private Partnerships (PPP) usually occurs at any time during the life of the project, causing large costs for the parties. Specifically, the toll road concessions are exposed to high demand risk. Usually, highway concession contracts incorporate a compensation mechanism in case of early termination. However, the traditional techniques of the Discounted Cash Flow (DCF) used to value infrastructure projects do not capture the irreversibility of the investment, the uncertainty and the mechanisms of compensation for risk that allow taking actions to the parties involved in a concession contract. The early termination of a toll road concession can be valued as the concessionaire's right to abandon the execution of the project. The objective of this document is to assess the mechanism of early termination of a toll road concession as a contingent asset, implementing the theory of real options, where the value of the 
abandonment option depends on the risk of demand. The valuation methodology is applied to a real case study of the Program of the Fourth Generation of Highway Concessions (4G) by Colombian Government. The valuation of early termination mechanisms can help to understand ex-ante the risks faced by the public and private parties of a highway concession contracts.

Key words: Real options; compensation mechanisms; early termination; Public-Private Partnerships.

JEL classification: G13, D86, H43, H54.

\section{Introducción}

La terminación anticipada de las asociaciones público-privadas (APP) es una situación anormal que ocurre con frecuencia. De acuerdo con la base de datos del Banco Mundial (2019), de 7125 APP adjudicadas en los países emergentes entre 1980 y 2018 , un total de 661 proyectos terminaron anticipadamente, lo cual representa alrededor del 9,27\% (The World Bank, 2019; Xiong, Zhang y Chen, 2015).

La terminación anticipada de una APP es una situación atípica, en donde el ciclo de vida esperado del proyecto se interrumpe por un evento que inviabiliza la ejecución de la APP; esta se puede dar por distintas razones, las cuales se pueden categorizar, según la parte responsable de la terminación, de la siguiente manera: a) responsabilidad de la entidad pública contratante, ante un incumplimiento de sus obligaciones; b) por mutuo acuerdo de las partes, ante el acontecimiento de un riesgo imprevisible, cambios de las condiciones de mercado o incumplimientos de ambas partes (pública y privada); c) por responsabilidad de la parte privada, producto de la incapacidad técnica, financiera u operativa; o d) ante una terminación unilateral por parte de la entidad pública, la cual puede consistir en una expropiación o en una recompra del proyecto, ya sea porque se desea nacionalizar el activo o porque la capacidad del proyecto es insuficiente para atender la demanda (Song, Hu y Feng, 2018).

Entre 1990 y 2018, Colombia ha adjudicado 46.696 millones de dólares en proyectos de APP, de los cuales el $53 \%$ han sido para el desarrollo de carreteras (The World Bank, 2019). Actualmente, Colombia se encuentra entre los diez países emergentes que más invierte a través del esquema de APP (The World Bank, 2019). Particularmente, entre 2014 y 2018, el Gobierno adjudicó más de 30 proyectos del programa de la Cuarta Generación de Concesiones Viales - 4G. 
Actualmente, dos concesiones de los 30 proyectos adjudicados del programa 4G terminaron de forma anticipada durante la fase de preconstrucción debido a que se sobrestimó la demanda de tráfico durante el proceso de planeación y no fue posible instalar las casetas de peaje. Estas dos concesiones se adjudicaron bajo la modalidad de iniciativa privada. Otras siete concesiones del programa $4 \mathrm{G}$ se encuentran en proceso de terminación anticipada por la activación de distintos tipos de riesgos.

La terminación anticipada de una APP genera: a) la suspensión de la construcción de la infraestructura y la prestación del servicio; b) grandes costos para el sector público además del tiempo que requiere volver a estructurar y adjudicar el proyecto y c) pérdidas para el concesionario y los financiadores de los proyectos (Xiong, Zhang y Chen, 2015).

Generalmente, la terminación anticipada de una APP lleva a disputas entre las partes como consecuencia de la negociación por el valor de la compensación que solicita la parte privada a cambio de devolver el activo de infraestructura a la parte pública. La compensación es la remuneración por la inversión y el esfuerzo realizado durante la ejecución del proyecto. La compensación no solo es importante para los accionistas del proyecto, sino también para los financiadores quienes han colocado una masa crítica de recursos para la construcción del proyecto.

El contrato de concesión 4G incorpora una fórmula para estimar el valor de la compensación por terminación anticipada en cualquier momento de la vida del proyecto. La terminación anticipada puede ser ejercida por el concedente, el concesionario o por mutuo acuerdo de las partes. La terminación anticipada de una concesión puede ser valorada como el derecho que tiene el concesionario a abandonar la ejecución del proyecto a cambio de una compensación por la inversión y el esfuerzo realizados.

La opción de terminar anticipadamente el proyecto se puede valorar como un activo contingente desde el momento de suscripción del contrato (ex-ante) en función del riesgo del proyecto, con el fin de identificar los estados de la naturaleza futura en los cuales se podría ejercer la posibilidad de terminar el proyecto y el valor que este tipo de flexibilidad gerencial le otorga a este.

El objetivo del presente documento es valorar el mecanismo de terminación anticipada de una concesión vial como un activo contingente, implementando la teoría de las opciones reales, donde el valor de la opción de abandono depende del riesgo de demanda, es decir, la evolución del tráfico. Bajo este contexto, se analizará la terminación anticipada para un caso particular: la liquidación 
anticipada del contrato por mutuo acuerdo. La metodología de valoración se aplicará a un estudio de caso real del programa de la Cuarta Generación de Concesiones Viales del Gobierno de Colombia bajo la modalidad de iniciativa privada, las cuales no cuentan con garantía de ingresos mínimos.

\section{Teoría de opciones reales}

La técnica del flujo de caja descontado (FCD) presenta cuatro principales deficiencias producto de sus supuestos: el primer supuesto del FCD se centra en que todos los flujos del proyecto deben ser descontados a una tasa. En general, es difícil determinar una tasa de descuento que refleje todos los riesgos no diversificables del proyecto y el costo de oportunidad del dinero en el tiempo en términos de una tasa conocida y constante a largo plazo. Esto supone que el capital siempre estará disponible al mismo costo, supuesto poco realista en un mundo sujeto a la incertidumbre (Hertz, 1964; Moreno Trujillo, 2015; Robichek y van Horne, 1967).

En segundo lugar, el FCD considera que el pronóstico que se realiza de los flujos de caja futuros es cierto y realizable, este supuesto no refleja la incertidumbre a la cual se encuentran expuestos los proyectos (Dixit y Pindyck, 1995; Hertz, 1964; Myers, 1984). En tercer lugar, el FCD no explica el efecto de diversificación, interdependencia y sinergia entre la cartera de proyectos de la firma (Mun, 2002; Myers, 1984; Trigeorgis y Mason, 1987). En cuarto lugar, el FCD subestima el valor del proyecto debido a que no considera los posibles cursos de acción de su gestión (flexibilidad gerencial) conforme se adquiere más información con el paso del tiempo. El FCD no valora el accionar gerencial, ni las decisiones subsecuentes (Copeland y Antikarov, 2001; Dixit y Pindyck, 1995; Kester, 1984; Myers, 1984; Trigeorgis y Mason, 1987). Por lo anterior, Myers (1984) considera que el FCD es una técnica que se debe usar para valorar activos relativamente seguros o sin incertidumbre.

Las opciones reales son el resultado de la búsqueda de más de medio siglo por subsanar las deficiencias de la técnica del FCD, particularmente enfocadas en capturar: a) la incertidumbre de los activos del sector real como resultado de los posibles estados de la naturaleza futuros (valoración de activos contingentes) y la revelación de información conforme pasa el tiempo; b) la irreversibilidad de la inversión, y c) el valor de los posibles cursos de acción en la gestión de los proyectos ante los distintos estados de la naturaleza futuros o la interdependencia entre el portafolio de proyectos de la firma, a lo cual Trigeorgis y Mason (1987) 
denominan la flexibilidad gerencial. Las opciones reales son la aplicación de la teoría de las opciones financieras para valorar proyectos del sector real y mejorar la toma de decisiones de inversión en la firma.

Una opción real es el derecho, pero no la obligación, de tomar una acción (diferir, expandir, contraer, abandonar, entre otras) sobre el proyecto o activo del sector real $\left(S_{T}\right)$ a un predeterminado precio de ejercicio $(K)$, en un tiempo establecido $(T)$ o en cualquier momento menor o igual al momento establecido (T) (Copeland y Antikarov, 2001). La identificación de ese "derecho" es equivalente a toda oportunidad, opción o curso de acción por parte de la gerencia, a lo cual denominamos flexibilidad gerencial.

La posibilidad de terminar anticipadamente un contrato de concesión puede ser valorada a través de la teoría de las opciones reales asumiendo que su comportamiento es similar a una opción de abandono, donde la parte privada (el concesionario) tiene la opción o el derecho de terminar anticipadamente el proyecto cuando el valor de compensación o liquidación es mayor al valor de continuar el proyecto (valor presente de los flujos futuros). Este ámbito de aplicación ha cobrado gran relevancia en las últimas cuatro décadas, donde se identifica un aumento significativo de la teoría de opciones reales. Particularmente, su impulso ha estado fundamentado en las limitaciones que presenta el análisis tradicional de valoración, entre estos, la técnica del valor presente neto (VPN).

Robichek y van Horne (1967) analizan la importancia de calcular el valor de la opción de abandono ante las deficiencias de las técnicas del FCD, para lo cual determinan como regla de decisión la opción de continuar o abandonar el proyecto; para valorar la opción utilizan la técnica de simulación de Monte Carlo. Dyl y Long (1969) y Bonini (1977) mejoran el modelo de valoración desarrollado por Robichek y van Horne.

Kensinger (1980) valora una opción de abandono para un periodo específico (put europea) a través del método de Black and Sholes. Myers y Madj (1983, 2001) valoran una opción de abandono (put americana) enfocada a una empresa que utiliza dos tipos de tecnología; para valorar la opción de abandono implementan la técnica de diferencias finitas. Huang y Chou (2006) estudian el caso del proyecto del tren de alta velocidad de Taiwán para valorar una garantía de ingreso mínimo y la opción de abandonar el proyecto, la cual se puede ejercer únicamente durante la fase de preconstrucción (opción europea).

Caselli, Marciante y Gatti (2009) diseñan una cláusula de indemnización para un contrato de una autopista por APP, lo cual permite a los sponsors abandonar el proyecto en una única fecha (opción europea) en la etapa de operación. 
Blank, Samanez, Baidya y Días (2016) analizan un proyecto hipotético de una autopista con una garantía mínima de tráfico, una garantía de tráfico máximo y una opción implícita de abandono.

Cabero, Sánchez y Lara (2016) estudian la compensación por parte del sector público (concedente) a la parte privada (concesionario) en proyectos de autopistas por APP cuando se produce la insolvencia del proyecto como consecuencia de un tráfico menor al esperado en la etapa de operación, la decisión de abandono se produce cuando el valor del proyecto es inferior a la deuda.

Igrejas, Cordeiro y Brandão (2017) estudian un proyecto de infraestructura de banda ancha, para el cual desarrollan un modelo de opciones reales a fin de valorar las inversiones secuenciales integradas a una opción de abandonar el proyecto durante la etapa de construcción (opción compuesta de invertir o abandonar). Liu, Gao y Cheah (2017), a través de opciones reales valoran los mecanismos de fijación de precios de la terminación anticipada de una autopista en fase de operación en dos diferentes escenarios: los flujos de caja excesivamente altos y los flujos de caja excesivamente bajos, utilizando una opción put y call americanas; la decisión de abandono se centra en el flujo de caja esperado del proyecto, asumiendo que si dicho valor se ubica por debajo de cierto porcentaje, el concesionario abandonará el proyecto y, en caso de que el valor del proyecto sea superior a un porcentaje, el Gobierno lo terminará unilateralmente.

Buso, Dosi y Moretto (2019), a través de la implementación de un modelo económico en tiempo continuo, estudian una opción de abandono (opción put) a favor de la parte privada, en caso de que el proyecto presente pérdidas, y un mecanismo de subsidio directo para la inversión de capital (opción call), que otorga el Gobierno. El análisis se centra en un problema de información oculta y el costo que asume el Gobierno al garantizar la continuidad del servicio en caso de una salida anticipada del contratista.

\subsection{Análisis de la fórmula de terminación anticipada}

Con el objetivo de determinar el precio del ejercicio $(K)$ de la opción de terminación anticipada del proyecto debemos conocer cuál es la compensación que resultaría de terminar el contrato de concesión de manera anticipada. En el contrato de concesión $4 \mathrm{G}$ el valor de la compensación por terminación anticipada es definido como el "valor de liquidación anticipada". La fórmula de liquidación anticipada del contrato de concesión estándar 4G establece que la terminación anticipada se puede presentar en cualquier momento del ciclo de vida del 
proyecto por tres circunstancias: a) por responsabilidad del concesionario; b) por responsabilidad del concedente; c) por mutuo acuerdo (fuerza mayor o caso fortuito), y d) por terminación unilateral del contrato de concesión producto de las facultades exorbitantes del Estado. De acuerdo con el contrato de concesión 4G, la terminación anticipada puede ocurrir durante cualquier momento del proyecto, sea la fase de preconstrucción, construcción u operación y mantenimiento.

El presente análisis se limitará a estudiar la terminación anticipada por mutuo acuerdo. La fórmula del contrato de concesión establece que el valor de liquidación anticipada será el resultado de la siguiente fórmula (Agencia Nacional de Infraestructura, 2016):

$$
V L_{m}=\sum_{h=1}^{m}\left[\left(A R_{h}-R_{h}\right) *\left(\frac{I P C_{m}}{I P C_{h}}\right) *(1+T E)^{(m-h)}\right]
$$

donde:

- $V L_{m}$ : valor de liquidación anticipada en el año $m$.

- $A R_{h}$ : corresponde a todos los gastos en que incurrió el concesionario para el desarrollo del proyecto en el año $h$.

- $R_{h}$ : corresponde al valor que recibió el concesionario como retribución por concepto de ingresos de peajes durante el año $h$.

- $I P C_{m}$ : inflación en el año $m$.

- $I P C_{h}$ : inflación en el año $h$.

- TE: corresponde a una tasa de descuento real, la cual reconoce el costo de oportunidad de los recursos de capital invertidos en el proyecto.

- $h$ : cada uno de los años desde la suscripción del contrato hasta el año $m$.

- $m$ : año en que ocurre la terminación anticipada del Contrato.

De acuerdo con la fórmula anterior, definiremos el precio de ejercicio $(K)$ de la opción de terminar anticipadamente el proyecto, en donde $K=V L$.

La fórmula de liquidación anticipada del contrato de concesión $4 \mathrm{G}$ se estructuró con el espíritu de brindar seguridad a los prestamistas en caso de una terminación anticipada del proyecto, permitiéndoles recuperar el saldo de la deuda. A través de los resultados del estudio de caso se analizará si efectivamente el valor de liquidación protege a los financiadores. 


\subsection{Definición de la opción de terminación anticipada}

La opción de terminar anticipadamente puede ser definida como el derecho a abandonar el proyecto que tiene la parte privada (concesionario) cuando el valor de liquidación anticipada $\left(V L_{m}\right)$ es mayor al valor presente del flujo de caja futuro $\left(V P F C_{m}\right)$ en el año $(m)$ :

$$
\text { Valor de la Opción }= \begin{cases}0 & \text { si } V L_{m}<V P F C_{m} \\ V L_{m}-V P F C_{m} & \text { si } V L_{m}>V P F C_{m}\end{cases}
$$

La opción puede ser valorada como una opción put americana con posición larga para el concesionario. Asumiremos que en todos los estados en donde $V L_{m}>V P F C_{m}$ se debe a un evento de fuerza mayor y, por tanto, ante la decisión racional del concesionario de abandonar el proyecto en esos estados de naturaleza, la entidad concedente acepta la terminación por mutuo acuerdo.

\subsubsection{El riesgo de demanda}

Los proyectos de concesiones viales tienen distintas fuentes de riesgo, los riesgos endógenos del proyecto pueden ser gestionados por la parte privada a través de distintas estrategias: la especialización en la gestión, la diversificación y el aseguramiento. No obstante, los riesgos exógenos del proyecto no pueden ser controlados por el sector privado y, generalmente, el mercado no ofrece productos para asegurarse contra este tipo de riesgos. El riesgo de demanda es el principal factor de incertidumbre exógeno del proyecto; del flujo de caja futuro dependen la posibilidad de financiación del proyecto, el pago de los gastos operacionales y la rentabilidad del concesionario (Cabero et al., 2016; Liu et al., 2017; Sánchez, Lara y Cabero, 2018).

En países en vía de desarrollo, las partes involucradas en una APP padecen de un sesgo optimista en la estimación de los flujos de caja futuros del proyecto debido a que el originador del proyecto, sea este la entidad pública o el sector privado (en caso de las iniciativas privadas), en ocasiones considera que sus expectativas positivas o alcistas sobre los ingresos futuros se realizarán, por lo que sobrestima los ingresos de tráfico. El sesgo es producto de la necesidad o la conveniencia de que el proyecto efectivamente se adjudique a una firma del sector privado y que esta, a su cuenta y riesgo, lo ejecute. Este fenómeno genera que el riesgo de demanda sea transferido completamente al sector privado, con 
una alta probabilidad de que regrese a la entidad concedente en forma de mayores riesgos o grandes costos (Bain, 2009; Ibrahim, Price y Dainty, 2006; Sánchez et al., 2018). Tratar de transferir el riesgo de demanda al concesionario puede resultar en la terminación del proyecto, ya que el concesionario no se encuentra en capacidad de soportar o absorber dicho riesgo.

Adicionalmente, los proyectos de APP requieren de altos niveles de endeudamiento que van entre el 70 y el $95 \%$ del capital del proyecto (Engel, Fischer y Galetovic, 2010). Estos grados de apalancamiento únicamente se logran a través del marco del Project Finance, el cual es viable solo en la medida en que la magnitud y volatilidad de los flujos futuros generados por la iniciativa sean estables, predecibles y con baja volatilidad. Los flujos de caja futuros del proyecto constituyen la garantía principal de los acreedores, lo que expone a los prestamistas a riesgo específicos del proyecto que pueden ser difíciles de diversificar (Sorge, 2004; Yescombe, 2014). Por lo tanto, una APP es viable únicamente en la medida en que sea posible conseguir los recursos para su financiación en el mercado.

Por todo lo anterior, se valorará la opción de terminar anticipadamente el proyecto en función del riesgo de demanda o riesgo de tráfico. Para modelar el riesgo de demanda se implementará el modelo de Brandao y Saraiva (2007) el cual asume que el tráfico o los ingresos de una concesión siguen un proceso de difusión conforme al Movimiento Browniano Geométrico (MBG).

\subsubsection{Modelo de tráfico}

A través de un proceso de Ito se puede obtener en tiempo discreto la fórmula del MBG, la cual se puede representar en periodos anuales, en función del valor del periodo anterior, de la siguiente manera:

$$
S_{t+1}=S_{t}\left\{\left(\alpha-\frac{1}{2} \sigma^{2}\right) t+\sigma \sqrt{t Z}\right\}
$$

donde:

$S_{t}$ : cambios incrementales del tráfico en un periodo de tiempo de un año.

$\alpha$ : tasa de crecimiento del tráfico en un año.

$\sigma$ : volatilidad del tráfico, la cual es constante.

$\sqrt{t Z}$, donde $Z \sim N(0,1)$. 
Para caracterizar la incertidumbre del tráfico futuro del proyecto se debe determinar un valor inicial del tráfico $\left(S_{t}\right)$, su tasa de crecimiento $(\alpha)$ y su volatilidad $(\sigma)$; estos datos de entrada se pueden definir a partir del estudio de la serie histórica de tráfico. El MBG asume que la tasa de crecimiento del tráfico $(\alpha)$ y su volatilidad $(\sigma)$ son constantes durante el ciclo de vida del proyecto.

\subsection{Método de valoración de la opción}

El precio y ejercicio de la opción de terminación anticipada será determinado a través del método de valoración binomial de Cox, Ross y Rubinstein (1979), integrando la técnica de simulación de Monte Carlo. El árbol binomial se construirá con intervalos de un año para los periodos en los cuales $V L_{m}>0$. El árbol binomial nos permitirá observar gráficamente los distintos estados de la naturaleza del activo subyacente y el ejercicio de la opción. Se realizarán distintas iteraciones para cada uno los nodos del árbol del activo subyacente. La simulación nos permitirá obtener el valor esperado de la opción y el VPN expandido del proyecto.

\section{Caso de estudio: concesión Buga-Buenaventura}

Aplicaremos el modelo propuesto de valoración para determinar el valor de la opción de terminación anticipada de la concesión Buga-Buenaventura, este proyecto fue estructurado y adjudicado en el marco del Programa de Concesiones Viales de Cuarta Generación (4G) del Gobierno de Colombia. La concesión Buga-Buenaventura tiene por objeto el diseño, la construcción, el mejoramiento, la operación y la administración de la doble calzada entre Buga y Buenaventura. Adicionalmente, el proyecto tiene por alcance la construcción de dos túneles nuevos, y la operación y el mantenimiento de 16 túneles existentes.

El contrato de concesión del proyecto vial Buga-Buenaventura se suscribió en el año 2016, entre la concesión Vía al Puerto S.A.S. y la Agencia Nacional de Infraestructura (ANI). El corredor Buga-Buenaventura se encuentra localizado en el departamento del Valle del Cauca, en el occidente del país (figura 1), es una arteria vial estratégica para el comercio exterior e interior debido a que Buenaventura es la principal zona portuaria de Colombia sobre el Pacífico, moviliza el 21,8\% del total de la carga portuaria del país (sin considerar carbón a granel) y transporta el $97,5 \%$ del total de la carga a través del Océano Pacífico (Superintendencia de Transporte, 2018). 
La concesión Buga-Buenaventura tiene una longitud de 111,3 km distribuida en cinco unidades funcionales ${ }^{1}$ (UF). El proyecto tiene un plazo de 30 años. El ciclo de vida esperado del proyecto se divide en las siguientes fases: un año de Fase de preconstrucción, cuatro años de fase de construcción y 25 años de fase de operación y mantenimiento (figura 2$)^{2}$. El proyecto cuenta con dos casetas de peajes: el peaje Loboguerrero, el cual se empieza a recaudar desde el acta de inicio del proyecto, y el peaje Calima, el cual inicia su recaudo a partir del segundo año del proyecto.

Figura 1: Ubicación geográfica proyecto Buga-Buenaventura

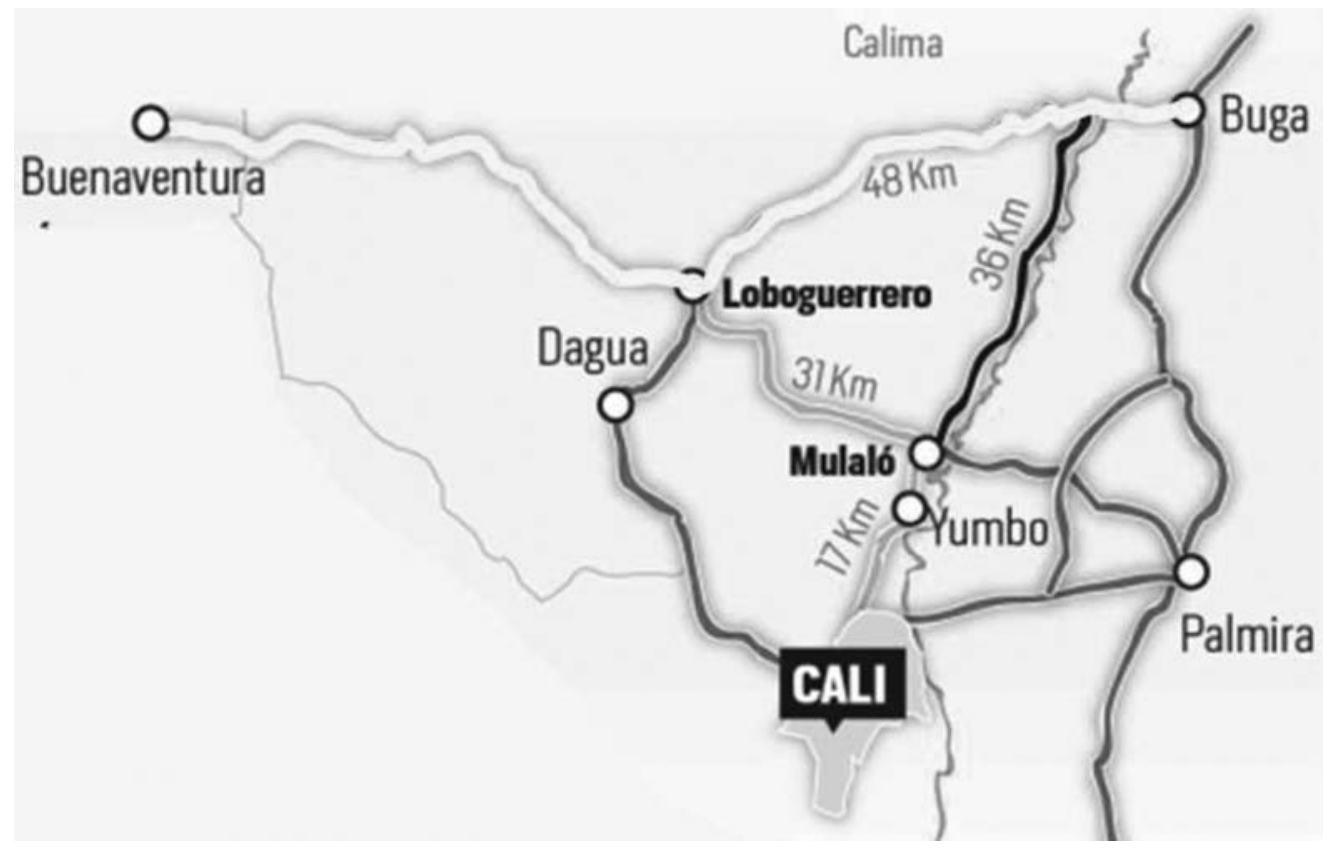

Fuente: concesión Vía al Puerto (2014).

1 "Unidad Funcional de Infraestructura: conjunto de estructuras de ingeniería e instalaciones indispensables para la prestación de servicios con independencia funcional, la cual le permitirá funcionar y operar de forma individual cumpliendo estándares de calidad y niveles de servicio para tal unidad, relacionados con la satisfacción de la necesidad que sustenta la ejecución del Proyecto de Asociación Público-Privada" (Presidencia de la Republica de Colombia, 2012).

2 El contrato de concesión estándar 4G agrega las fases de preconstrucción y construcción, en una categoría denominada "Etapa preoperativa", y a la fase de operación, mantenimiento y reversión, la considera la "Etapa operativa". 
Figura 2: Ciclo de vida esperado de la concesión Buga-Buenaventura

Plazo del proyecto: 30 años

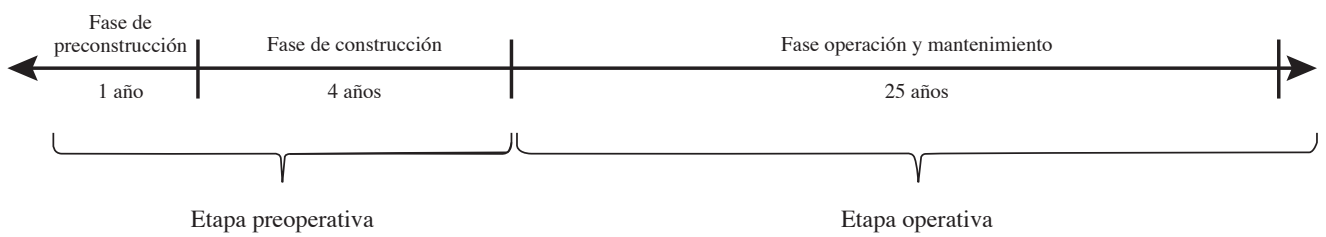

Fuente: elaboración propia con datos de Agencia Nacional de Infraestructura (2016).

La concesión Buga-Buenaventura fue adjudicada a través de la modalidad de iniciativa privada. En Colombia, las iniciativas privadas son una modalidad de las APP, a través de la cual el sector privado es el originador de una propuesta para la ejecución de un proyecto de APP. Las iniciativas privadas tienen tres objetivos principales: a) que el sector privado identifique las oportunidades en el mercado para el desarrollo de APP con objetivo económico y social; b) que a través de la planeación y estructuración del proyecto por parte del sector privado se logre la viabilidad técnica, financiera y social, y c) que el sector privado retenga mayores riesgos del proyecto.

En vista de que la concesión Buga-Buenaventura es una APP de iniciativa privada, el concesionario retiene mayores riesgos, particularmente en relación con la demanda, ya que no cuenta con dos garantías fundamentales que el Programa $4 \mathrm{G}$ ofreció para los contratos de concesión por iniciativa pública: a) no cuenta con peaje sombra o recursos del presupuesto público (vigencias futuras), y b) no tiene garantías de tráfico (diferencial de recaudo), denominada en la literatura académica garantía de ingreso mínimo. Por lo anterior, los ingresos del concesionario únicamente dependen del tráfico y la tarifa de peaje regulada en el contrato. Para fines académicos asumiremos que el proyecto tiene un plazo total de 30 años, sin la posibilidad de solicitar ninguna clase de prórroga adicional en caso de disminución de la demanda de tráfico.

\subsection{Datos y parámetros del proyecto}

De acuerdo con las obligaciones técnicas, prediales, socioambientales y financieras establecidas en el contrato de concesión, se definió un CAPEX (capital expenditure) por un valor de $\$ 735.809$ millones, todas las unidades funcionales (UF) del proyecto se construirán entre el año 2 y el año 5. Se asumió un OPEX (Operational expenditures) por la suma de \$1.105.214 millones. Los precios de 
referencia del contrato de concesión se encuentran expresados en pesos constantes de 2015. El flujo de caja incorpora una cuenta de reserva de OPEX equivalente al $50 \%$ del gasto del siguiente año y una cuenta de reserva de mantenimientos periódicos del $50 \%$ del gasto por dicho rubro para los siguientes 3 años. Se asume una inflación constante del $4 \%$ durante la vida del proyecto. Se supone una depreciación del total del CAPEX del $4 \%$ anual, durante la etapa operativa del proyecto, por el método de línea recta. Se asume una tasa efectiva de tributación sobre la utilidad antes de impuestos equivalente al $25 \%$. De acuerdo con la curva zero cupón de los TES del Gobierno colombiano con vencimiento a un año para el 2015, se determinó una tasa libre de riesgo del 6,51 \% (Banco de la República, 2019).

Acorde con el equity establecido en el contrato de concesión y la estructura de financiación estándar del mercado para proyectos $4 \mathrm{G}$, se asume una relación $30 \%$ de equity y $70 \%$ de deuda. Un costo de oportunidad del equity del $14 \%$ y un costo de financiación equivalente al $12 \%$, el cual, por practicidad, incluye todos los fees producto de la estructuración financiera del crédito sindicado. Para la financiación se asume un crédito sindicado con una única línea senior, por un plazo máximo de 20 años y posibilidad de amortización acelerada, con un periodo de gracia de 5 años durante la etapa preoperativa. Los intereses de la financiación durante la construcción se capitalizan. De acuerdo con la estructura de capital se determinó un WACC del 9,98\%.

Se asume un contrato de crédito con tres principales obligaciones por parte del concesionario (Covenants): a) durante la vida del préstamo no se pagarán dividendos a los accionistas; b) se supone una amortización de la deuda por el método esculpido (sculped method) para proyectos financiados bajo el marco del Project Finance y c) el ratio de cobertura del servicio de la deuda objetivo $\left(D S C R_{o}\right)$ durante la vida del crédito será igual a $2 x$. El ratio de cobertura del servicio de la deuda para cada año $t\left(D S C R_{t}\right)$ se puede definir de la siguiente manera:

$$
D S C R_{t}=\frac{F C S D_{t}}{S D_{t}}
$$

donde:

$F C S D_{t}$ : flujo de caja disponible para atender el servicio de la deuda en el año $t$. $S D_{t}$ : servicio de la deuda en el año $t$. 
De acuerdo con Gatti (2013), el $D S C R_{t}$ de un proyecto de transporte puede estar entre 1,35x-1,5x. En Colombia, los créditos sindicados en pesos de las concesiones $4 \mathrm{G}$ establecen un $D S C R_{t}$ entre $1,45 \mathrm{x}-1,5 x$. Dado que el flujo de caja no considerara cuenta de reserva del servicio de la deuda, determinamos un $D S C R_{o}$ un poco mayor, con el fin de suponer que ese margen adicional $(0,5 x)$ haga las veces de cuenta de reserva del servicio de la deuda.

De acuerdo con lo anterior, la amortización en el año $t\left(A_{t}\right)$ se esculpirá (sculped method) en función del $D S C R_{o}$, lo cual quiere decir que la amortización será equivalente al valor que mantenga siempre el $D S C R_{o}=2 x$, en otras palabras, el flujo de caja final de cada periodo durante la vida del crédito siempre será igual al servicio de la deuda del periodo. La forma en la que se calcula la amortización se puede representar como:

$$
A_{t}=\frac{F C S D_{t}}{D S C R_{o}}-i_{t}
$$

donde:

$F C S D_{t}$ : flujo de caja disponible para atender el servicio de la deuda en el año $t$. $D S C R_{o}$ : ratio de cobertura del servicio de la deuda objetivo en el año $t$. $i_{t}$ : interés del crédito que se debe pagar en el año $t$.

Esta regla de amortización acelera el pago del saldo de la deuda, si el flujo de caja disponible para atender el servicio de la deuda $F C S D_{t}$ es más de dos veces el servicio de la deuda en el periodo $\left(S D_{t}\right)$.

La amortización propuesta nos permite identificar en los distintos escenarios que modelaremos si el valor de terminación del contrato de concesión cubre el saldo acumulado de la deuda en cada periodo en cada año $(t)$. Así pues, si nos encontramos en el escenario de tráfico bajo, el proyecto se amortizará en un periodo de tiempo mayor que si nos encontramos en el escenario de tráfico alto, esto nos permitirá observar la cobertura de la fórmula del valor de liquidación anticipada del contrato de concesión en los distintos escenarios del tráfico (escenario alto, medio y bajo).

Para la caracterización del proceso probabilístico que sigue el tráfico, se utilizó la serie histórica mensual de tráfico por categorías del peaje Loboguerrero entre el 01 de enero de 2001 y el 31 de diciembre de 2015. De acuerdo con la serie de tráfico se determinó el $T P D A_{t}$ para cada año entre 2001 y 2015; a partir 
de esta información se determinaron los retornos logarítmicos de $\frac{T P D A_{t+1}}{T P D A_{t}}$, lo cual permitió determinar una tasa de crecimiento del tráfico $(\alpha)$ de $3,18 \%$ y una volatilidad del tráfico $(\sigma)$ de $4,80 \%$. Se asume como tráfico inicial $\left(S_{0}\right)$ el TPDA del año 2015.

La tasa de crecimiento promedio del tráfico proyectado para el escenario medio del peaje Loboguerrero en el Estudio de Tráfico y Demanda del proyecto fue de 3,12 \% (Concesión Vía al Puerto, 2014), una diferencia de 6 puntos básicos con respecto al resultado de la tasa de crecimiento del tráfico $(\alpha)$ obtenido a través del análisis de la serie de tiempo del tráfico entre 2001-2015. Para objeto de la modelación del tráfico asumiremos la tasa obtenida a través de la serie de tiempo.

Con los parámetros $S_{0}, \alpha y \sigma$ es posible caracterizar el proceso de difusión del tráfico, en tiempo discreto, para un escenario de crecimiento medio, utilizando la ecuación del MBG, el cual iteraremos a través de la técnica de simulación de Monte Carlo. Por razones de practicidad, modelaremos el total del tráfico de los dos peajes en función del tráfico de Loboguerrero. A partir del estudio de tráfico y demanda se pudo observar que en promedio el tráfico del peaje Calima es equivalente al $64,71 \%$ del tráfico del peaje Loboguerrero.

Por último, para estimar la tarifa $(\bar{X})$ de peaje se tomó la tarifa por categorías establecida en el contrato de concesión para los años 2016-2019 (a partir del año 2019 esta se debe actualizar con la inflación). El contrato define una tarifa por categorías única para ambos peajes. Así pues, la tarifa $(\bar{X})$ es el resultado de calcular la tarifa por categoría para cada tipo de vehículo $\left(x_{i}\right)$ definida en el contrato de concesión, ponderada por el porcentaje de tráfico promedio por categoría $\left(w_{i}\right)$. El porcentaje de tráfico promedio por categoría $\left(w_{i}\right)$ se determinó a partir del tráfico porcentual anual estimado por categorías del peaje Loboguerrero en el Estudio de Tráfico y Demanda.

\subsection{Resultados del modelo}

El flujo de caja determinístico dio como resultado un VPN (sin flexibilidad) por valor de \$435.689 millones y una tasa interna de retorno (TIR) nominal por la suma de $13,31 \%$ (tabla 1). Las necesidades de recursos de capital iniciales para la construcción del proyecto fueron por valor de $\$ 926.918$ millones $^{3}$, el préstamo

3 Cifras en millones de pesos corrientes. 
se repaga en un periodo de 12 años. El valor presente de los ingresos de peajes $(\mathrm{VPIP})^{4}$ para el caso base sin incertidumbre es equivalente a $\$ 1,78$ billones.

Tabla 1: Escenario determinístico

\begin{tabular}{|c|c|c|c|c|c|}
\hline Escenario/Indicador & VNP & TIR $(\%)$ & VPIP & Capital inicial & Vida del crédito (años) \\
\hline Escenario determinístico & $\$ 435,689$ & 13,31 & $\$ 1.784,830$ & $\$ 926.918$ & 12 \\
\hline
\end{tabular}

Fuente: elaboración propia.

\subsubsection{Flujo de caja probabilístico (escenarios alto, medio y bajo)}

Para el pronóstico del flujo de caja probabilístico se definieron tres escenarios de tráfico, en donde el valor de tráfico inicial $\left(S_{0}=5.145\right)$ y la volatilidad $(\sigma=4,80 \%)$ se mantuvieron constantes en los tres escenarios. Los tres escenarios se diferencian con respecto al nivel de la tasa de crecimiento promedio del tráfico $(\alpha)$. El escenario medio supone una tasa de crecimiento del tráfico $(\alpha)$ igual a $3,18 \%$, equivalente a la media de los retornos logarítmicos de la serie de tiempo de tráfico del corredor vial. Para los escenarios medio y alto la tasa de crecimiento del tráfico $(\alpha)$ se aumentó en $50 \%$ y se disminuyó en $50 \%$, respectivamente.

El flujo de caja probabilístico del escenario bajo $(\alpha=1,59$ y $\sigma=4,80 \%)$ dio como resultado un VPN esperado de $\$ 73.214$ millones, con un nivel de confianza del $90 \%$ entre $-\$ 321.871$ y $\$ 537.631$ millones. La probabilidad de pérdida es del $41,87 \%$. La TIR nominal esperada es del $10,41 \%$. El VPIP esperado fue de $\$ 1,54$ billones. Las necesidades de recursos de capital fueron de \$940.991 millones, el préstamo se repaga en un periodo de 13 años.

El flujo de caja probabilístico del escenario medio dio como resultado un VPN esperado de $\$ 450.950$ millones, con un nivel de confianza del $90 \%$ entre -\$40.265 millones y $\$ 1.036 .640$ millones. La probabilidad de pérdida es del $6,87 \%$. La TIR nominal esperada es del 13,24\%. El VPIP esperado fue de $\$ 1,79$ billones. Las necesidades de recursos de capital fueron de $\$ 927.574$ millones, el préstamo se repaga en un periodo de 12 años (figura 3 ).

4 El VPIP es una medida del contrato estándar de las concesiones $4 \mathrm{G}$, la cual consiste en expresar el total de los ingresos de recaudo de peajes en precios constantes de referencia del Contrato de Concesión, descontados por el WACC del proyecto. Para el caso particular del Proyecto Buga-Buenaventura, los precios de referencia del contrato están dados en pesos colombianos a 31 de diciembre del año 2015. El VPIP permite comparar los distintos escenarios de tráfico.

5 La probabilidad de que el proyecto arroje pérdidas o no sea viable es equivalente a toda probabilidad en donde el VNP sin flexibilidad sea menor que 0 . 
Figura 3: VPN y TIR, escenarios bajo, medio y alto
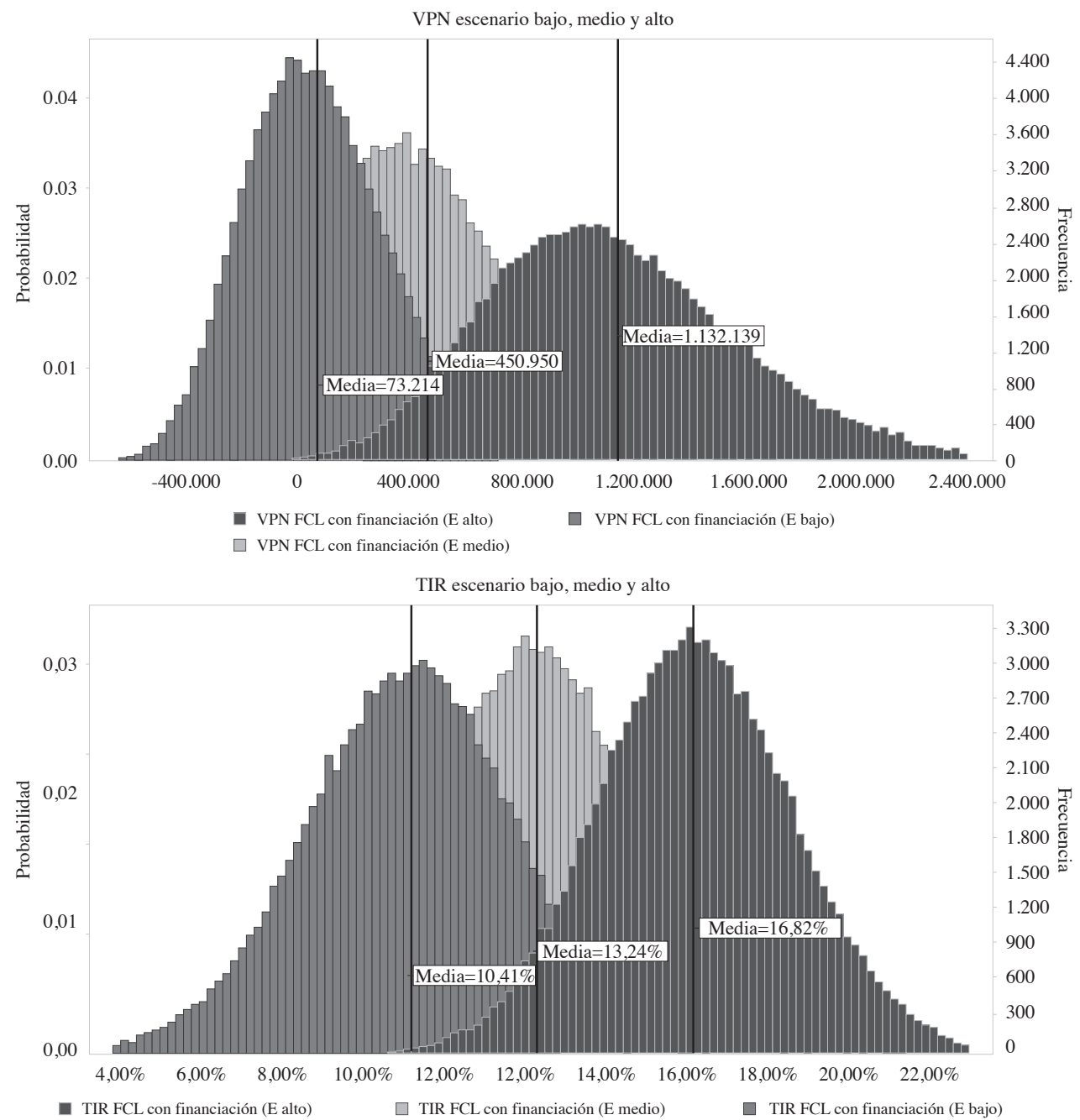

Fuente: elaboración propia con uso de Crystal Ball.

El flujo de caja probabilístico del escenario alto $(\alpha=4,77$ y $\sigma=4,80 \%)$ dio como resultado un VPN esperado de $\$ 1.132 .139$ millones, con un nivel de confianza del $90 \%$ entre $\$ 461.851$ millones y $\$ 1.946 .987$ millones. La probabilidad de pérdida es del $0,05 \%$; la TIR nominal esperada es del 16,82\%; el VPIP esperado fue de $\$ 2,22$ billones. Las necesidades de recursos de capital fueron de $\$ 908.479 \mathrm{mi}$ llones, el préstamo se repaga en un periodo de 11 años. En la tabla 2 se presenta un cuadro comparativo con los resultados de los tres escenarios. 
Tabla 2: Cuadro comparativo flujo de caja probabilístico

\begin{tabular}{|l|c|c|c|c|c|c|c|c|}
\hline Escenario & $5 \%$ & VNP & $95 \%$ & VPN $<0(\%)$ & TIR $(\%)$ & VPIP & Capital inicial & Crédito (años) \\
\hline Bajo & -321.871 & 73.214 & 537.631 & 41,87 & 10,41 & 1.546 .907 & 940.991 & 13 \\
\hline Medio & -40.265 & 450.950 & 1.036 .640 & 6,87 & 13,24 & 1.794 .894 & 927.574 & 12 \\
\hline Alto & 461.851 & 1.132 .139 & 1.946 .987 & 0,05 & 16,82 & 2.228 .817 & 908.479 & 11 \\
\hline
\end{tabular}

Fuente: elaboración propia.

\subsubsection{Volatilidad del proyecto}

De acuerdo con el modelo de volatilidad de Copeland y Antikarov (2001), con un nivel de confianza del $90 \%$, el proyecto tiene una volatilidad esperada de $27,87 \%$ en un intervalo entre 27,29 y $28,11 \%$. La volatilidad se estimó a través del procedimiento de análisis de remuestreo (boostrap) con 10.000 pruebas. Se tomará la volatilidad del proyecto $\left(\sigma_{p}=27,87 \%\right)$ para el cálculo del valor de la opción de terminar el proyecto anticipadamente en todos sus escenarios. En la figura 4 se presenta la función de frecuencia acumulada de la volatilidad.

Figura 4: Volatilidad del proyecto

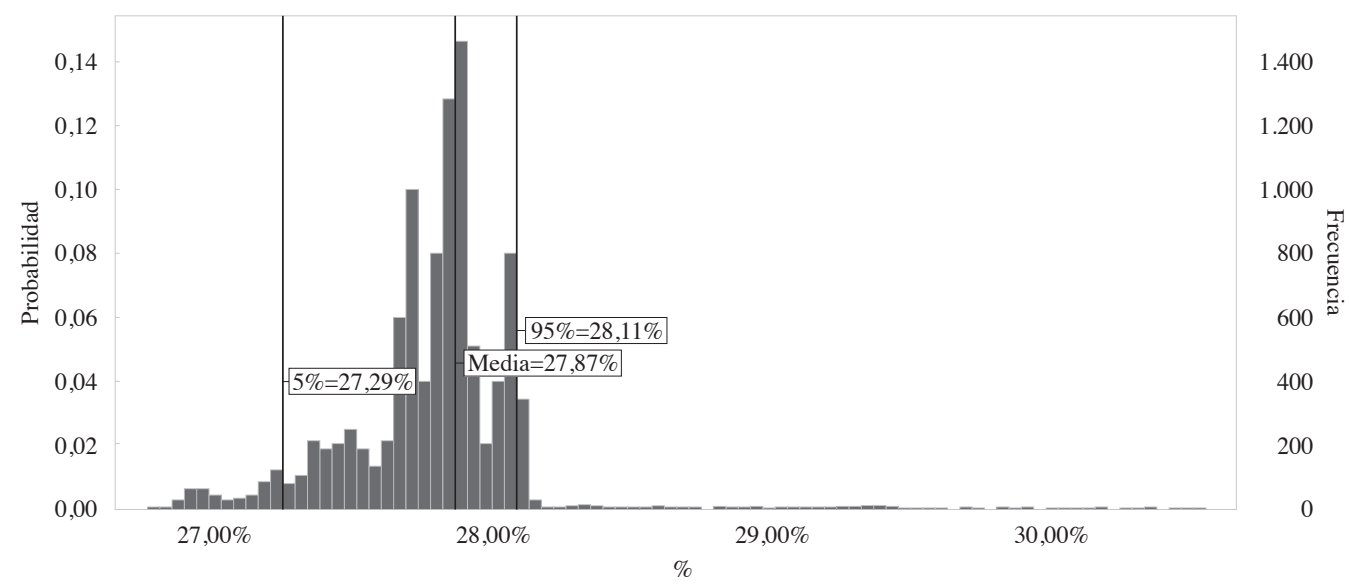

Fuente: elaboración propia con uso de Crystal Ball.

\subsubsection{Valor de liquidación anticipada por mutuo acuerdo}

El valor de liquidación $\left(V L_{h}\right)$, tomando para su cálculo una $T E$ igual a la tasa establecida en el contrato para terminación por mutuo acuerdo de las partes, la cual es de $8,13 \%$ anual, inferior al WACC supuesto para el proyecto en 1,85\%, 
arrojó que en el escenario de tráfico bajo, el concesionario tiene incentivos para terminar anticipadamente el contrato de concesión hasta el año 16. En contraposición, en el escenario de tráfico alto, el concesionario tiene incentivos para terminar anticipadamente el proyecto hasta el año 11. En el escenario de tráfico medio, el valor de liquidación va hasta el año 13. En los tres escenarios, los resultados de la fórmula de liquidación anticipada del contrato de concesión cubren exitosamente el saldo de la deuda más intereses. En la figura 5 se observa el valor de liquidación $\left(V L_{h}\right)$, por mutuo acuerdo con $T E=8,13 \%$ y el saldo de la deuda más intereses para cada uno de los escenarios.

Figura 5: Valor de liquidación anticipada vs. saldo de la deuda más intereses

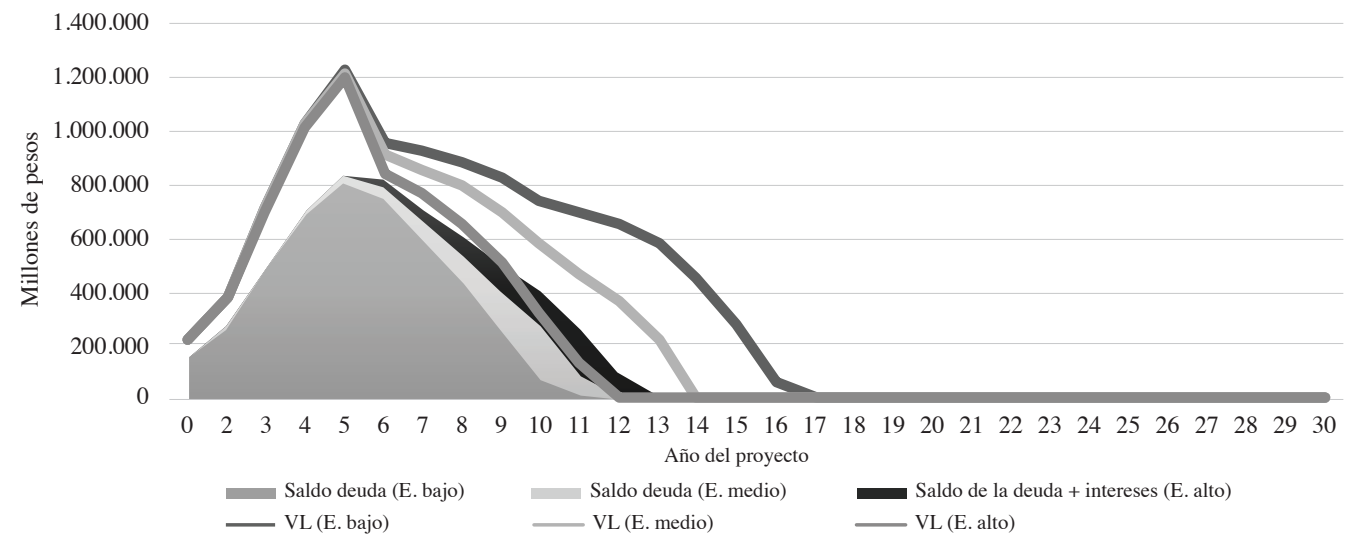

Fuente: elaboración propia.

\subsubsection{Valoración de la opción: terminación anticipada por mutuo acuerdo}

Al utilizar el valor de liquidación anticipada del contrato de concesión para cada año $\left(V L_{h}\right)$, como precio de ejercicio de la opción de abandono, tomando para su cálculo la $T E$ definida en el contrato de concesión para terminación por mutuo acuerdo, el valor de la opción de terminar anticipadamente el proyecto en el escenario determinístico dio como resultado la suma de $\$ 471.103$ millones y un VPN expandido de $\$ 906.792$ millones.

En el escenario de tráfico bajo, la opción de terminar anticipadamente el proyecto tiene un valor esperado de $\$ 833.901$ millones y un VPN expandido con media de $\$ 907.115$ millones, con un intervalo de confianza del $90 \%$, tomando valores entre $\$ 899.476$ y $\$ 941.164$ millones. La opción de abandono se ejercerá con una probabilidad del $100 \%$ en los años 5 al 9, último año de la fase de construcción y primeros años de la fase de operación y mantenimiento. 
En el escenario de tráfico medio, la opción de terminar anticipadamente el proyecto dio como resultado un valor esperado de $\$ 507.516$ millones y un valor esperado del VPN expandido de $\$ 958.466$ millones, con un intervalo de confianza del $90 \%$, tomando valores entre \$888.132 y \$1.207.025 millones. La opción de abandono tiene una probabilidad del $83 \%$ de ser ejercida en el año 5 (último año de construcción).

En el escenario de tráfico alto, la opción de terminar anticipadamente el proyecto dio como resultado un valor esperado de $\$ 179.315$ millones y un valor esperado del VPN expandido de $\$ 1.311 .454$ millones, con un intervalo de confianza del $90 \%$, tomando valores entre $\$ 895.600$ y $\$ 1.986 .992$ millones. La opción de abandono tiene una probabilidad del $50 \%$ de ser ejercida en el año 5. En la figura 6 se presenta el gráfico de superposición entre el VPN sin flexibilidad y el VNP expandido, y el gráfico de la probabilidad de ejercicio de la opción, para los tres escenarios.

Figura 6: vNP sin flexibilidad $v s$. VPN expandido

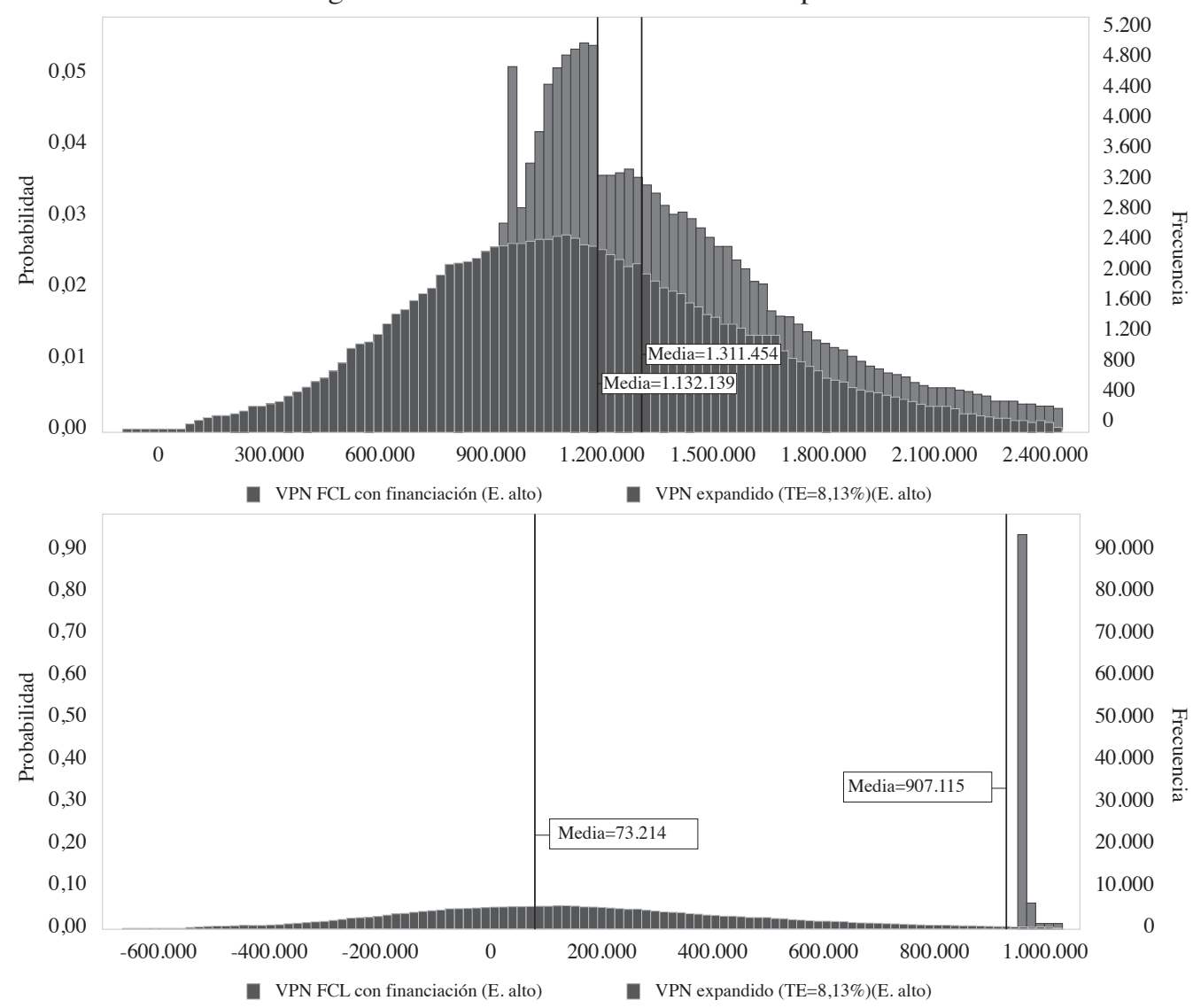




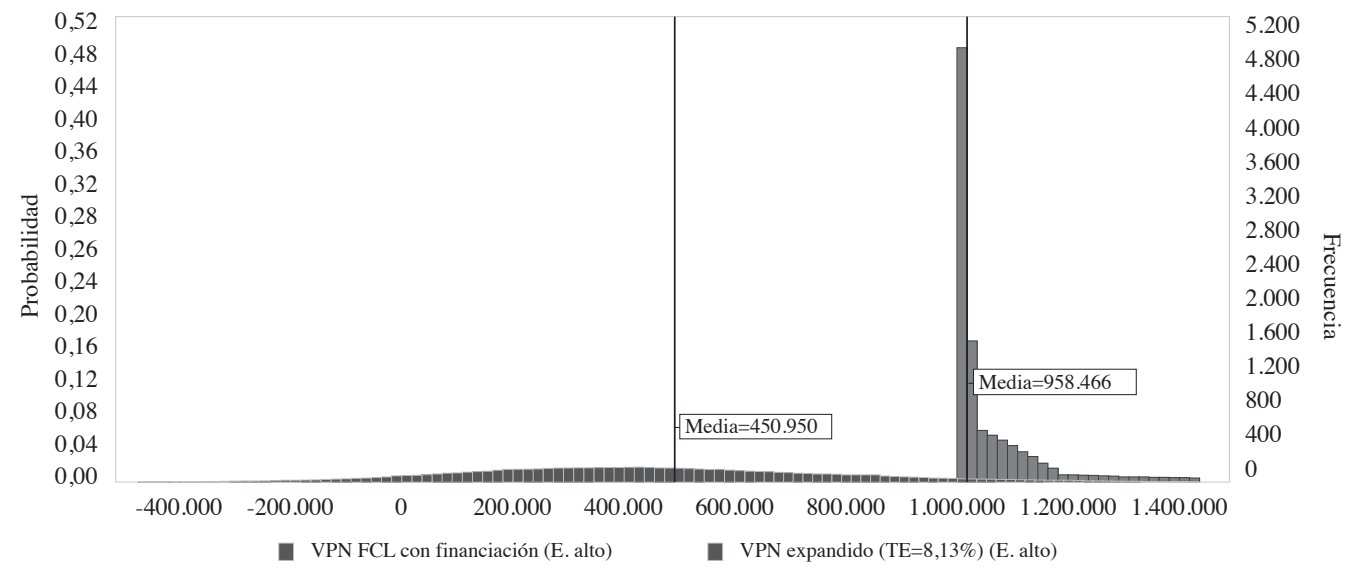

Fuente: elaboración propia con uso de Crystal Ball.

Figura 7: Probabilidad de ejercicio de la opción

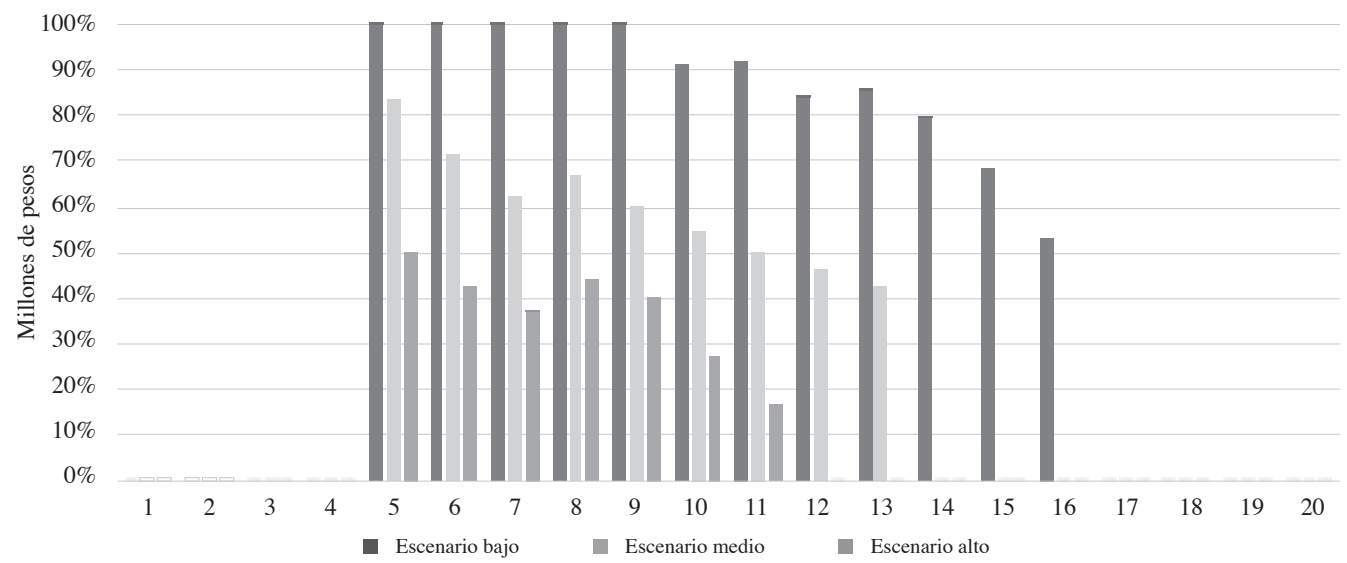

Fuente: elaboración propia.

En la tabla 3 se presenta un resumen comparativo de los resultados del valor de la opción de terminar anticipadamente el proyecto y el VPN expandido en los tres escenarios de tráfico. 
Tabla 3: Valor de la opción y VPN expandido

\begin{tabular}{|l|c|c|c|c|c|c|c|c|}
\hline \multirow{2}{*}{ Escenario } & \multicolumn{3}{|c|}{ Valor esperado opción real } & \multicolumn{2}{c|}{ Valor esperado VPN expandido } & \multicolumn{2}{c|}{$\begin{array}{c}\text { Max. probabilidad de } \\
\text { ejercicio de la opción }\end{array}$} \\
\cline { 2 - 9 } & $5 \%$ & Media & $95 \%$ & $5 \%$ & Media & $95 \%$ & Año & $\%$ \\
\hline Bajo & 403.527 & 833.901 & 1.221 .326 & 899.476 & 907.115 & 941.164 & 5 & 100 \\
\hline Medio & 170.379 & 507.516 & 928.369 & 888.132 & 958.466 & 1.207 .025 & 5 & 83 \\
\hline Alto & 40.004 & 179.315 & 433.728 & 895.600 & 1.311 .454 & 1.986 .992 & 5 & 50 \\
\hline
\end{tabular}

Fuente: elaboración propia.

\subsubsection{Análisis de sensibilidad de la volatilidad}

De acuerdo con el análisis de sensibilidad de la volatilidad frente al valor de la opción de terminar el proyecto anticipadamente se evidencia que en el escenario de tráfico bajo el valor de la opción varía muy poco; con una volatilidad del $10 \%$ el valor de la opción es de $\$ 852.439$ millones y con una volatilidad del $90 \%$ el valor es de $\$ 860.527$ millones. Por el contrario, en el escenario de tráfico alto el valor de la opción varía sustancialmente ante un cambio en la volatilidad; con una volatilidad del $10 \%$ la opción tiene un valor de $\$ 10.062$ millones y con una volatilidad del $90 \%$ la opción tiene un valor de \$598.861 millones. En la figura 8 se presenta el análisis de sensibilidad de la volatilidad frente al valor de la opción en los tres escenarios.

Figura 8: Análisis de sensibilidad de la volatilidad frente al valor de la opción

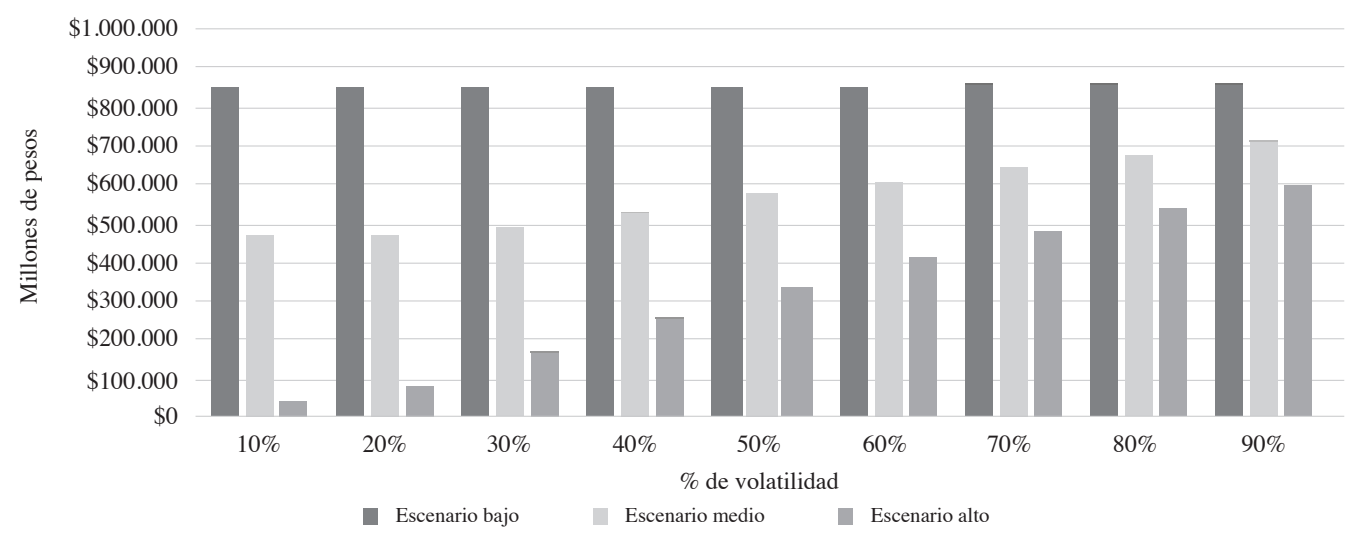

Fuente: elaboración propia. 


\section{Conclusiones}

Las concesiones viales por iniciativa privada en Colombia se encuentran particularmente expuestas al riesgo de demanda. El tráfico es la única fuente de ingresos de las concesiones por iniciativa privada. Resulta imposible acertar en el pronóstico a largo plazo de los escenarios futuros del tráfico. De acuerdo con distintos análisis, se ha demostrado que generalmente las partes suelen sobrestimar las proyecciones futuras de ingresos, y este riesgo suele volver a la parte concedente a un gran costo.

El tráfico de una carretera concesionada se encuentra particularmente expuesto a cambios en las condiciones de la demanda, los cuales son exógenos al control y gestión del concesionario. Los ingresos futuros son un factor crítico en la viabilidad financiera del proyecto. No obstante, las técnicas tradicionales del FCD utilizadas para valorar proyectos de infraestructura no capturan la irreversibilidad de la inversión, la incertidumbre y los mecanismos de compensación por riesgo que permiten tomar acciones a las partes intervinientes de un contrato de concesión.

A través de la teoría de las opciones reales fue posible valorar como un activo contingente la cláusula de terminación anticipada incorporada en los contratos de concesión 4G. La posibilidad de abandonar la concesión en cualquier momento, a cambio de una compensación, otorga un gran valor al proyecto y limita las pérdidas, en cualquiera de los escenarios analizados. En particular, para el escenario de tráfico bajo, la opción de terminación anticipada actúa como una garantía de ingreso mínimo, retornando el riesgo de demanda al concedente, lo cual evita la posibilidad de costosas renegociaciones y largos litigios.

De acuerdo con los resultados del estudio de caso, la opción de terminar el proyecto anticipadamente nunca se ejerce durante la fase de preconstrucción y los primeros tres años de la fase de construcción. No obstante, el último año de construcción (año 5) es el momento más crítico del proyecto, dado que se han invertido todos los recursos en la construcción de la infraestructura del corredor vial y no se ha recibido a cambio el total de la retribución. Particularmente, en el año 5 del proyecto, el método de valoración implementado captura la irreversibilidad de la inversión, la incertidumbre en el largo plazo y la posibilidad de abandonar el proyecto. En el último año de construcción, en el escenario bajo, la opción de abandonar el proyecto se ejerce con un $100 \%$ de probabilidad y en el escenario alto con un $50 \%$ de probabilidad. 
Se evidenció que la fórmula de liquidación anticipada en distintos escenarios siempre es superior al saldo de la deuda más los intereses. La fórmula de liquidación actúa como una garantía de última instancia para proteger a los prestamistas del proyecto en caso de un escenario de tráfico bajo o, incluso, ante la liquidación anticipada por fuerza mayor en cualquier escenario de tráfico.

A pesar de que el proyecto no cuente con garantías de ingreso mínimo, la fórmula de terminación anticipada del contrato de concesión $4 \mathrm{G}$ para iniciativas privadas actúa como un mecanismo de compensación por riesgo en los escenarios de tráfico bajo, esta garantía implícita hace viable la financiación del proyecto y protege al concesionario y a los financiadores de una posible insolvencia por baja demanda.

La aplicación de la metodología de valoración por opciones reales puede ayudar a comprender de manera ex-ante los riesgos a los cuales se enfrentan las partes pública y privada de un contrato de concesión, así como a valorar los mecanismos contractuales para mitigar o distribuir el riesgo entre las partes, y al diseño de nuevos mecanismos que permitan redactar contratos más eficientes.

\section{Referencias}

Agencia Nacional de Infraestructura (2016). Contrato de concesión bajo el esquema de APP n. ${ }^{\circ} 003$ de 2016. Parte General y especial. Bogotá D.C.: Agencia Nacional de Infraestructura. Recuperado de https://www.contratos.gov.co/consultas/detalleProceso.do?numConstancia $=16-20-1375$

Ashuri, B., Asce, M., Kashani, H., Molenaar, K. R., Asce, M., Lee, S. y Lu, J. (2012). Risk-neutral pricing approach for evaluating вот highway projects with government minimum revenue guarantee options. Journal of Construction Engineering and Management, 138(4), 545-557. https://doi.org/10.1061/(ASCE)CO. 1943-7862.0000447.

Bain, R. (2009). Error and optimism bias in toll road traffic forecasts. Transportation, 36(5), 469-482. https://doi.org/10.1007/s11116-009-9199-7

Banco de la República (2019). Tasa cero cupón pesos. Recuperado de http://www.banrep. gov.co/es/tes

Blank, F. F., Samanez, C. P., Baidya, T. K. N. y Dias, M. A. G. (2016). Economic valuation of a toll road concession with traffic guarantees and the abandonment option. Production, 26(1), 39-53. https://doi.org/10.1590/0103-6513.168713 
Bonini, C. P. (1977). Capital investment under uncertainty with abandonment options. The Journal of Financial and Quantitative Analysis, 12(1), 39-54. https://doi. org/10.2307/2330286

Brandão, L. E. y Saraiva, E. C. (2007). Valuing government guarantees in toll road projects. Universidade Catolica do Rio de Janeiro, 1-27.

Buso, M., Dosi, C. y Moretto, M. (2019). Termination fees and contract design in publicprivate partnerships. SSRN Electronic Journal. https://doi.org/10.2139/ssrn.3313335

Cabero Colín, F., Lara Galera, A. y Sánchez Galera, A. (2016). Default and abandonment option in motorway concessions. Journal of Infrastructure Systems, 23(1), 1-9. https://doi.org/10.1061/(ASCE)IS.1943-555X.0000309

Caselli, S., Marciante, A. y Gatti, S. (2009). Pricing final indemnification payments to private sponsors in project-financed public-private partnerships: An application of real options valuation. Journal of Applied Corporate Finance, 21(3), 95-106. https:// doi.org/10.1111/j.1745-6622.2009.00243.x

Concesión Vía al Puerto (2014). Estudio de viabilidad volumen I: Estudio de tráfico y demanda proyecto Vía al Puerto - Corredor Vial Buga-Buenaventura. Bogotá D.C: Agencia Nacional de Infraestructura. Recuperado de ftp://ftp.ani.gov.co/Iniciativas Privadas/IP Via Puerto - Buga - Buenaventura/

Copeland, T. y Antikarov, V. (2001). Real options: A practitioner's guide. New York: ThOmSOn TEXERE.

Cox, J. C., Ross, S. A. y Rubinstein, M. (1979). Option pricing: A simplified approach. Journal of Financial Economics, 7(3), 229-263. https://doi.org/10.1016/0304405X(79)90015-1

Dixit, A. K. y Pindyck, R. S. (1995). The options approach to capital investment. En M. Press (ed.). Real Options and Investment under Uncertainty: Classical Readings and Recent Contributions (pp. 61-77). Cambridge: MIT Press.

Dyl, E. A. y Long, H. W. (1969). Abandonment value and capital budgeting: Comment. The Journal of Finance, 24(1), 88-95. https://doi.org/10.2307/2326129 
Engel, E., Fischer, R. y Galetovic, A. (2010). The economics of infrastructure finance: Public-private partnerships versus public provision. EIB papers, 15. Recuperado de http://www.econstor.eu/handle/10419/45373

Gatti, S. (2013). Project Finance in Theory and Practice. Project Finance in Theory and Practice. Oxford: Academic Press.

Hertz, D. B. (1964). Risk Analysis in capital investments. Harvard Business Review, 42, 95-106.

Huang, Y. y Chou, S. (2006). Valuation of the minimum revenue guarantee and the option to abandon in вОт infrastructure projects. Construction Management and Economics, 24(4), 379-389. https://doi.org/10.1080/01446190500434997

Ibrahim, A. D., Price, A. D. F. y Dainty, A. R. J. (2006). The analysis and allocation of risks in public private partnerships in infrastructure projects in Nigeria. Journal of Financial Management of Property and Construction, 11(3), 149-164. https://doi. org/10.1108/13664380680001086

Igrejas, R., Cordeiro, L. y Brandão, L. E. (2017). When is abandonment not an option? Dealing with PPP contract and government interests. Advances in Public-Private Partnerships - Proceedings of the 2nd International Conference on Public-Private Partnerships (pp. 486-498). https://doi.org/10.1061/9780784480267.038

Kensinger, J. W. (1980). Project abandonment as a put option: Dealing with the capital investment decision and operating risk using option pricing theory. Working paper, 20-21.

Kester, W. C. (1984). Today's options for tomorrow's growth. Harvard Business Review, 62, 153-160.

Liu, J., Gao, R. y Cheah, C. Y. J. (2017). Pricing mechanism of early termination of ppp projects based on real option theory. Journal of Management in Engineering, 33(6), 04017035. https://doi.org/10.1061/(ASCE)ME.1943-5479.0000556

Moreno Trujillo, J. F. (2015). Una introducción a las opciones reales. Bogotá D.C: Universidad Externado de Colombia.

Mun, J. (2002). Real Options Analysis. New Jersey: John Wiley y Sons. 
Myers, S. C. (1984). Finance theory and financial strategy. Interfaces, 14(1), 126-137. https://doi.org/10.1287/inte.14.1.126

Myers, S. C. y Majd, S. (1983). Calculating abandonment value using option pricing theory. Sloan School of Management Working Paper, Fist Draft (May), 1-38.

Myers, S. C. y Majd, S. (2001). Abandonment value and project life. Real Option and Investment under Uncertainty: Classical Regarding and Recent Contributions (pp. 295-312). Cambridge: The MIT Press.

Presidencia de la República de Colombia. Decreto 1467 de 2012, por el cual se reglamenta la Ley 1508 de 2012 (2012). Recuperado de http://wsp.presidencia.gov.co/Normativa/ Decretos/2012/Documents/Julio/06/dec146706072012.pdf

Robichek, A. A. y van Horne, J. C. (1967). Abandonment value and capital budgeting. The Journal of Finance, 22(4), 577-589.

Sánchez Soliño, A., Lara Galera, A. L. y Cabero Colín, F. (2018). Valuation of the option of early reversion in road concessions. European Journal of Transport and Infrastructure Research, 18(2), 239-249.

Song, J., Hu, Y. y Feng, Z. (2018). Factors Influencing Early Termination of PPP Projects in China. Asce Library, 34(1), 1-10. https://doi.org/10.1061/(ASCE)ME.19435479.0000572 .

Sorge, M. (2004). The nature of credit risk in project finance. BIS Quarterly Review, 91102. Recuperado de http://cendoc.esan.edu.pe/paginas/infoalerta/project/risk.pdf

Superintendencia de Transporte (2018). Boletín Estadístico: tráfico portuario en Colombia. Bogotá D.C: Superintendencia de Transporte. Recuperado de http://www. supertransporte.gov.co/

The World Bank (2019). Private participation in infrastructure database. Recuperado de http://ppi.worldbank.org/snapshots/region/latin-america-and-the-caribbean

Trigeorgis, L. y Mason, S. P. (2001). Valuing managerial flexibility. En The MIT Press (ed.). Real Option and Investment Under Uncertainty: Classical Regarding and Recent Contributions (pp.47-60). Cambridge: The MIT Press. 
Xiong, W., Zhang, X. y Chen, H. (2015). Early-termination compensation in public-Private partnership projects. Journal of Construction Engineering Management, 142(4), 1-9. https://doi.org/10.1061/(ASCE)CO.1943-7862.0001084.

Yescombe, E. R. (2014). Principles of Project Finance (2 ed.). Oxford: Elsevier. 